\title{
Editorial
}

\section{Estratégias de enfrentamento durante a pandemia da COVID-19 em uma instituição de ensino superior de Enfermagem}

$\mathrm{O}$ cenário imposto pela pandemia da COVID-19 exigiu que as universidades públicas brasileiras organizassem seus processos de trabalho para equacionar os problemas ou as situaçóes críticas e emergenciais desvelados por essa emergência sanitária mundial. A Escola Paulista de Enfermagem da Universidade Federal de São Paulo (EPE-Unifesp), no auge dos seus 81 anos de fundação, sentiu-se extremamente comprometida com essa realidade e buscou adotar estratégias que respondessem eficazmente às demandas de ensino, pesquisa, extensão, gestão e assistência.

Assim como no Brasil, em outros países do mundo ${ }^{(1)}$ os estudantes de Enfermagem foram suspensos das práticas assistenciais hospitalares e de extensão comunitária e, a partir dessa decisão, numerosas açóes foram compartilhadas em relação à formação profissional dos estudantes e às atividades que seriam realizadas para não comprometer o currículo acadêmico.

Nacionalmente, no ensino, inúmeras atividades foram planejadas para manter o vínculo com os estudantes, mesmo a distância, bem como para repensar o currículo dos cursos, de modo que garantissem o conhecimento e o compromisso social necessários para a formação de profissionais qualificados para o Sistema Único de Saúde (SUS). A EPE, seguindo orientaçôes da Pró-Reitoria de Graduação da Unifesp, adotou o regime de atividades domiciliares especiais como estratégia para assegurar o cumprimento das atividades teóricas. Para os estudantes do último ano do curso de graduação, optou-se pelo reinício das atividades práticas. Embora o risco de adquirir a COVID-19 durante a prática assistencial seja considerável, ${ }^{(2)}$ estudantes no mundo se preocupam com a extensão das consequências da pandemia e buscam propor açóes que minimizem impactos negativos em suas formaçóes. ${ }^{(3)}$ Dessa forma, o compromisso social da EPE em formar enfermeiros para o SUS num momento de tamanha emergência sanitária fez-nos planejar esse retorno, garantindo a máxima segurança possível.

Sobre as atividades de pesquisa, por incentivo público, foram destinados para o país recursos que contribuem com novos métodos de diagnóstico, tratamento e interrupção da transmissão relacionados ao combate da COVID-19. A iniciativa engloba pesquisas relacionadas à história natural da doença; o desenvolvimento e a avaliação de testes, de alternativas terapêuticas e de vacinas; a avaliação da atenção à saúde nos três níveis de complexidade diante da epidemia; o uso de equipamentos de proteção individual (EPI) nas 
ações de prevenção, controle e manejo; a adesão e o cumprimento das medidas de prevenção e controle; a gestão de serviços na pandemia, entre outros temas relacionados à doença. As linhas de pesquisa foram definidas a partir de diretrizes da Organização Mundial da Saúde (OMS), alinhadas às prioridades nacionais em discussão entre o Ministério da Saúde e especialistas de todo o país, considerando a necessidade de resposta rápida e investimentos em estudos mais promissores. ${ }^{(4)}$ Nossos docentes e pesquisadores responderam a essa demanda e submeteram diversos projetos pleiteando subsídios para a realização dos seus estudos, tendo como compromisso responder com ciência a essa pandemia (Quadro 1).

Quadro 1. Projetos de pesquisa elaborados durante a pandemia na EPE-Unifesp

\begin{tabular}{|c|c|}
\hline Projeto de pesquisa & Nível \\
\hline A prática do aleitamento materno na pandemia da COVID-19 & Graduação \\
\hline $\begin{array}{l}\text { Aconselhamento telefônico de enfermagem para pacientes com câncer e a evolução destes na } \\
\text { pandemia COVID-19: estudo retrospectivo }\end{array}$ & Pós-graduação Stricto Sensu \\
\hline $\begin{array}{l}\text { Análise da efetividade de mensagens de texto na adesão do uso de máscaras e distanciamento } \\
\text { social em tempos de pandemia }\end{array}$ & Pós-graduação Stricto Sensu \\
\hline $\begin{array}{l}\text { Análise e perfil da dor lombar em profissionais do âmbito hospitalar na época da pandemia } \\
\text { COVID-19 }\end{array}$ & Pós-graduação Stricto Sensu \\
\hline $\begin{array}{l}\text { Aspectos emocionais e sociais de profissionais da saúde em quarentena por suspeita ou } \\
\text { confirmação de infecção por COVID-19 }\end{array}$ & Pós-graduação Stricto Sensu \\
\hline $\begin{array}{l}\text { Associação de sinais de alerta precoce com as categorias da classificação de risco de pacientes } \\
\text { com suspeita ou confirmação de COVID-19 atendidos no serviço de emergência }\end{array}$ & Graduação \\
\hline Avaliação da qualidade de vida dos sobreviventes de sepse por COVID-19 em um hospital terciário & Pós-graduação Stricto Sensu \\
\hline $\begin{array}{l}\text { Avaliação das alterações bioquímicas e o prognóstico dos pacientes com COVID-19 utilizando as } \\
\text { cadeias de Markov }\end{array}$ & Pós-graduação Stricto Sensu \\
\hline $\begin{array}{l}\text { Avaliação de equipamentos de proteção individual para profissionais de saúde: revisões } \\
\text { sistemáticas }\end{array}$ & Pós-graduação Stricto Sensu \\
\hline $\begin{array}{l}\text { Avaliação do cuidado de enfermagem a pacientes com COVID-19 em hospitais universitários } \\
\text { brasileiros }\end{array}$ & Pós-graduação Stricto Sensu \\
\hline $\begin{array}{l}\text { Conhecimento, atitudes e prática de pacientes oncológicos em tratamento quimioterápico durante } \\
\text { a pandemia COVID-19 }\end{array}$ & Graduação \\
\hline $\begin{array}{l}\text { Criação de um centro multidisciplinar de desenvolvimento, prototipagem, otimização e validação de } \\
\text { equipamentos de proteção individual para sustentabilidade e segurança na saúde }\end{array}$ & Pós-graduação Stricto Sensu \\
\hline Cuidado multiprofissional em pacientes com COVID-19 & Graduação \\
\hline $\begin{array}{l}\text { Evidências da validade dos resultados de enfermagem em resposta à ventilação mecânica: } \\
\text { resposta ao desmame e classificação dos resultados de enfermagem em pacientes em estado } \\
\text { crítico por COVID-19 }\end{array}$ & Pós-graduação Stricto Sensu \\
\hline Evolução da prevalência da infecção da COVID-19 em gestantes: inquéritos sorológicos seriados & Pós-graduação Stricto Sensu \\
\hline Higiene oral em pacientes sob ventilação mecânica: validação de protocolo & Pós-graduação Stricto Sensu \\
\hline $\begin{array}{l}\text { Infecção pelo vírus SARS-CoV-2 em transplantados renais: vigilância em saúde, screening e } \\
\text { fatores de risco associados }\end{array}$ & Pós-graduação Stricto Sensu \\
\hline Lesão renal aguda pelo vírus SARS-CoV-2 em pacientes com COVID-19: revisão integrativa & Pós-graduação Stricto Sensu \\
\hline $\begin{array}{l}\text { Orientação de alta por aconselhamento telefônico em pacientes com doenças cardiovasculares - } \\
\text { alerta para prevenção COVID-19 }\end{array}$ & Pós-graduação Stricto Sensu \\
\hline $\begin{array}{l}\text { Orientação de manutenção de estabilidade para RNI por aconselhamento telefônico em pacientes } \\
\text { em uso de varfarina e prevenção de COVID-19 }\end{array}$ & Graduação \\
\hline Práticas e percepções de profissionais da oncologia na pandemia da COVID-19 & Graduação \\
\hline $\begin{array}{l}\text { Prevenção de sintomas e lesões osteomusculares em profissionais de saúde durante a pandemia } \\
\text { da COVID-19: elaboração e validação de uma planilha }\end{array}$ & Pós-graduação Stricto Sensu \\
\hline Recomendações da RCP em paciente com COVID-19 & Pós-graduação Stricto Sensu \\
\hline Saúde materno-infantil entre mulheres infectadas por COVID-19 no período perinatal & Pós-graduação Stricto Sensu \\
\hline $\begin{array}{l}\text { Saúde mental de profissionais de enfermagem do Brasil durante a pandemia da COVID-19: estudo } \\
\text { transversal }\end{array}$ & Pós-graduação Stricto Sensu \\
\hline $\begin{array}{l}\text { Cartilha de orientação sobre os cuidados com a pele aos profissionais de saúde na pandemia da } \\
\text { COVID-19 }\end{array}$ & $\begin{array}{c}\text { Pós-graduação Stricto Sensu e } \\
\text { Graduação }\end{array}$ \\
\hline
\end{tabular}

$\mathrm{Na}$ extensão, as atividades de interação entre a comunidade e a universidade foram redirecionadas de modo que a fusão do conhecimento popular com o científico garantisse o intercâmbio de novos conhecimentos e am- 
pliasse o envolvimento da sociedade com o combate relacionado à transmissibilidade do coronavírus e açóes de promoção da saúde. ${ }^{(5)}$ Estudantes e docentes participaram de campanhas de imunização para a sociedade, bem como desenvolveram, por meio de recursos tecnológicos, estruturas que permitissem maior aproximação dos indivíduos com a sociedade, visando dirimir prejuízos psicossomáticos reportados pela população durante o período de isolamento social.

A EPE, diante do cenário apresentado, mobilizou-se e, no cumprimento da sua função gerencial, implementou, em parceria com as áreas de liderança da Unifesp (Pró-Reitoria de Administração e Gestão de Pessoas), o processo de trabalho remoto para os seus Servidores Docentes e Técnicos Administrativos. Foram realizadas escalas de trabalho que permitiram flexibilizar a linha operacional, tática e estratégica da instituição. Docentes e Técnicos Administrativos em Educação que não faziam parte do grupo de risco (profissionais acima de 60 anos, hipertensos, asmáticos, diabéticos e fumantes) participaram das atividades extensionistas programadas, bem como do estágio curricular supervisionado para os estudantes da $4^{a}$ série do curso de Graduação de Bacharelado em Enfermagem e com atividades domiciliares especiais com as três outras séries. As atividades acadêmicas da pós-graduação stricto sensu foram mantidas de forma remota. A equipe operacional foi dividida de modo que propiciou que todas as atividades administrativas ocorressem normalmente. Importantes medidas de segurança sanitária, físicas e eletrônicas foram inclusas como procedimento operacional padrão em meio à pandemia. Fisicamente, a administração da EPE aderiu ao uso obrigatório de máscara nas dependências da unidade universitária e foram instaladas barreiras de proteção na recepção e faixas de sinalização que pedem o distanciamento de $1,5 \mathrm{~m}$ de indivíduo para indivíduo. Dispensers de álcool em gel foram instalados e houve aumento considerável relacionado ao número de vezes em que as áreas comuns foram higienizadas, bem como passou-se a mensurar a temperatura individual de quem utiliza as dependências da escola. É fato que a diretoria da EPE-Unifesp enfrentou inúmeros desafios na condução dos seus processos de trabalho, todavia os métodos emergenciais chamados de "gestâo de crise" adotados permitiram que, minimamente, a estrutura organizacional fosse mantida com o objetivo de assegurar a condução dos processos de trabalho.

$\mathrm{Na}$ assistência, medidas importantes foram potencializadas para garantir a segurança dos profissionais e estudantes e ampliar a dinâmica alinhada ao relacionamento e à integraçáo das equipes. O que já era uma constância na prática profissional e estudantil tornou-se indispensável e fundamental para o exercício do cuidar. Os envolvidos nas atividades do Hospital São Paulo/Hospital Universitário (HSP/HU) e ambulatórios, vinculados à EPE-Unifesp, realizaram treinamentos presenciais e a distância, que tiveram como objetivo capacitar os enfermeiros e técnicos de enfermagem contratados pelo $\mathrm{HSP} / \mathrm{HU}$ da Unifesp emergencialmente para assistir os pacientes com COVID-19. 
A EPE-Unifesp, a partir do cenário apresentado, implementou inúmeras iniciativas de modo que se viabilizassem as atividades de ensino, pesquisa, extensão, gestáo e assistência já programadas. Para orientar a comunidade estudantil, as famílias dos estudantes e dos colaboradores, os grupos interligados e os profissionais da saúde, em especial os da área de enfermagem, os departamentos que compóem a EPE-Unifesp construíram documentos digitais (fluxogramas, histórias em quadrinhos, cartazes educativos, entre outros), mobilizaram os estudantes para a realizaçáo da campanha de vacinação contra o vírus influenza $(\mathrm{H} 1 \mathrm{~N} 1$ e $\mathrm{H} 2 \mathrm{~N} 3)$ para idosos, pessoas em condiçôes especiais e militares, estimularam e implementaram treinamentos específicos para os profissionais que se mantiveram ativos durante a pandemia em suas atividades assistenciais, em especial aos auxiliares, técnicos e enfermeiros do HU ligado à EPE-Unifesp. Além disso, fomentaram importantes pesquisas em parcerias com instituiçôes governamentais, sob incentivo público, para contribuir com o desenvolvimento de novas descobertas durante o período de isolamento social e o pico da transmissáo do vírus SARS-CoV-2. Cabe ressaltar a campanha realizada acerca da doação de EPI, bem como o fornecimento de utensílios pelo HU/HSP-Unifesp.

A pandemia de COVID-19 tem ressaltado de maneira comovente o papel essencial que enfermeiras, enfermeiros e outros profissionais de saúde desempenham para proteger a saúde das pessoas e salvar vidas. Um novo relatório da OMS, intitulado "The State of the World's Nursing 2020" (O Estado da Enfermagem no Mundo 2020), pede mais investimentos em educação, condiçôes de trabalho e liderança para profissionais de enfermagem, fortalecendo suas contribuiçóes aos sistemas de saúde. ${ }^{(6)}$ Nesse contexto, muitas atividades ainda estão sendo previstas, mas com a certeza de que a contribuiçáo social e o aprendizado adquirido no transcurso dessa pandemia serão permanentes e imprimirão um novo modo de ser e fazer enfermagem na EPE.

\section{Agradecimentos}

Agradecemos ao Especialista Bruno Henrique Sena Ferreira pela contribuição intelectual para a realização do editorial.

Profa. Dra. Janine Schirmer Escola Paulista de Enfermagem, Universidade Federal de São Paulo, São Paulo, SP, Brasil. https://orcid.org/0000-0003-0783-2961

Prof. Dr. Alexandre Pazetto Balsanelli Escola Paulista de Enfermagem, Universidade Federal de São Paulo, São Paulo, SP, Brasil. https://orcid.org/0000-0003-3757-1061 


\section{Como citar:}

Schirmer J, Balsanelli AP. Estratégias de enfrentamento durante a pandemia da COVID-19 em uma instituição de ensino superior de Enfermagem. Acta Paul Enferm.

DOI: http://dx.doi.org/10.37689/acta-ape/2020EDT0006

\section{Referências}

1. Pather N, Blyth P, Champman JA, Dayal MR, Flack NAMS, Fogg QA, et al. Forced Disruption of Anatomy Education in Australia and New Zealand: An Acute Response to the Covid-19 Pandemic. Anat Sci Educ. 2020;13(3):284-97.

2. Bernstein L, Boburg S, Sacchetti M, Brown E. Covid-19 hits doctors, nurses and EMTs, threatening health system [March 17, 2020]. [Internet]. [cited 2020 Jul 17]. Available from: https://www.washingtonpost. com/health/covid-19-hits-doctors-nurses-emts-threatening-health-system/2020/03/17/f21147e867aa-11ea-b313-df458622c2cc_story.html.

3. Dewart G, Corcoran L, Lorraine T, Kristin P. Nursing education in a pandemic: Academic challenges in response to COVID-19. Nurse Educ Today. 2020;92:104471.

4. Siqueira SM, Jesus VS, Santos EN, Whitaker MC, Sousa BV, Camargo CL. Extension activities, health promotion and sustainable development: the experience of a nursing research group. Esc Anna Nery. 2017;21(1):e20170021.

5. Brasil. Ministério da Saúde. Governo do Brasil destina R\$ 50 milhões para pesquisas sobre coronavírus [Internet]. Brasília (DF): Ministério da Saúde; 2020. [citado 2020 Jul 17]. Disponivel em: https://www. saude.gov.br/noticias/agencia-saude/46634-governo-do-brasil-destina-r-50-milhoes-para-pesquisassobre-coronavirus.

6. Organização Mundial da Saúde (OMS). Em meio à pandemia de COVID-19, novo relatório da OMS pede investimento urgente em profissionais de enfermagem [Internet]. Brasilia (DF): OMS; 2020. [citado 2020 Jul 17]. Disponivel em: https://www.paho.org/bra/index.php?option=com_ content\&view=article\&id=6139:em-meio-a-pandemia-de-covid-19-novo-relatorio-da-oms-pedeinvestimento-urgente-em-profissionais-de-enfermagem\&ltemid=812. 